\title{
Chromosomal Microarray Genetic Testing - A Qualitative Investigation among Parents of Children with Autism Spectrum Disorders in Rural North Carolina
}

\section{Xu L ${ }^{1 *}$, Sun $L^{2}$, Mitchell $L C^{3}$, Richman $A R^{1}$ and Floyd $A E^{1}$}

${ }^{1}$ Department of Health Education and Promotion, College of Health and Human Performance, Greenville, East Carolina University, Greenville, NC, 27858, USA ${ }^{2}$ Department of Communication Sciences and Disorders, College of Allied Health Sciences, Greenville, East Carolina University, Greenville, NC, 27834, USA ${ }^{3}$ Department of Human Development and Family Science, College of Health and Human Performance, East Carolina University, Greenville, NC, 27858, USA

\begin{abstract}
Background: Although Chromosomal Microarray (CMA) genetic testing is currently officially recommended as a part of the routine care in the Autism Spectrum Disorders diagnostic process, little is known about parents emotional responses regarding taking their children to undergo CMA genetic testing. To ensure adequate access to and utilization of genetic technologies and dispel the concerns regarding CMA, it is critical to understand parents' emotional responses to this test.

Method: We conducted qualitative interviews using a purposive sampling technique with 45 parents of children with ASD in rural eastern North Carolina. This geographical location maintains one of the highest rates of families who are Medicaid-eligible and underserved parents of children with ASD in North Carolina.

Results: We audio-taped all interviews, transcribed them verbatim, and conducted content analysis to identify themes and subthemes. Approximately $37 \%$ of our participants had more than one child with autism. Almost half of our parents (42.8\%) were ethnic minorities and all Hispanic parents $(26.3 \%)$ either had no health insurance or were Medicaid-eligible. Our preliminary results demonstrate that negative emotions, such as fear or guilt might be the factors most likely associated with parents' decisions regarding taking their children to undergo CMA testing.

Conclusion: The most salient research finding is that over half of the participants $(58 \%)$ reported having negative emotions toward CMA. Among these negative emotions, the feeling of fear, worry, anxiety, self-blaming and stress were the most frequently reported responses regardless parents' educational level, income and ethnicity. Pretesting counseling that addresses parents' negative emotions would be in urgent need to dispel parents' concerns related to CMA.
\end{abstract}

Keywords: Chromosomal microarray (CMA) genetic testing; Parents' perceptions; Qualitative study; In-depth interviews; Emotional responses

\section{Introduction}

Autism spectrum disorder (or ASD), constitutes a wide range of developmental conditions featured by impaired social development and interaction, as well as disrupted patterns of behaviors [1]. Rather than occurring as a single explicable abnormality, each child with ASD has unique presentations of the disorder and faces different challenges with communication, social skills and behaviors, potentially throughout a lifetime [2]. Although the clear etiology of ASD is still elusive, genetic factors, such as variants within genes, defects in associated copy number variants, or CNVs, as well as epigenetic signaling, have been clearly demonstrated as strong components in the disorder's development [3-5].

Due to the important role genetic factors play in the possible causes of ASD, clinical genetic evaluation, such as genetic tests and counseling, have the potential to bring benefits for parents and families of children with ASD [6]. Among existing genetic tests associated with ASD, Chromosomal Microarray Analysis (CMA) has been recently endorsed as a first-tier test by the American College of Medical Genetics owing to its improved detection of genomic abnormalities [7]. Data from CMA genetic testing holds a variety of implications for both autism research and management at the discretion of the tested child's parents $[5,8]$. As indicated by the International Standard Cytogenomic Array Consortium, the discovery of copy number variants through CMA testing may be stored in a database for future access and further analysis [4]. This and other types of genotypic information allow for the proposal of new candidate genes which may advance etiological knowledge. In additional, a more specific clinical diagnosis reveals the pathology of the child's disorder, allowing families access to a wider range of services such as behavioral, family support and medical services for their children [6,9]. The identification of known genetic pathways in the diagnosis may also uncover a more accurate recurrence risk of ASD in future children of both parents, which may help with informed decisions on family planning [10].

Despite the potential benefits of genetic testing, research indicates a gap in understanding the motivators and barriers pertaining to utilizing genetic testing services among parents of children with ASD $[6,11]$. While previous research has found that although the majority parents of children with ASD demonstrated favorable attitudes toward autism genetic testing, perceived

*Corresponding author: $\mathrm{Xu} \mathrm{L}, \mathrm{PhD}$, Department of Health Education and Promotion College of Health and Human Performance, East Carolina University, 300 Curry Court, Greenville, NC 27858, USA, Tel: (252) 328-1611; Fax: (252) 328-1285; E-mail: xul14@ecu.edu

Received September 13, 2016; Accepted November 10, 2016; Published November 17, 2016

Citation: Xu L, Sun L, Mitchell LC, Richman AR, Floyd AE (2016) Chromosoma Microarray Genetic Testing - A Qualitative Investigation among Parents of Children with Autism Spectrum Disorders in Rural North Carolina. J Clin Med Genomics 4 143. doi: $10.4172 / 2472-128 X .1000143$

Copyright: @ $2016 \mathrm{Xu} \mathrm{L}$, et al. This is an open-access article distributed unde the terms of the Creative Commons Attribution License, which permits unrestricted use, distribution, and reproduction in any medium, provided the original author and source are credited. 
consequences such as genetic discrimination, incurred costs, and apprehension surrounding the results might lead to a decline to testing [10-15]. We conducted a systematic literature review on emotional factors and autism genetic testing and found that none of the seventeen included articles had explored how emotional factors might affect parents' decisions on taking their children to undergo CMA genetic testing [16]. To better utilize the existing genetic tests and services, there is an immediate need to understand what emotional factors might affect parents' decisions associated with CMA genetic testing-one of the new generation of whole-genome sequencing.

This pilot study sought to investigate parental awareness and attitudes of CMA genetic testing, particularly focusing on how emotions might affect parents' decision-making process in taking their children to undergo the test. Our study sought to 1) explore parents' perceptions of CMA genetic testing; 2) analyze parents' responses to identify emotion-related motivations or barriers towards involving their children in CMA testing. Results of this study will inform the development of a larger scale survey and subsequent educational interventions among parents of children with ASD.

\section{Method}

\section{Study sample}

Prior to implementation, study protocol and procedures were approved by the Institutional Review Board (IRB) at East Carolina University. Our research team was composed of two autism researchers and one community member who was bilingual (English/Spanish) and had extensive experience working with Hispanic families with children affected by ASD.

A snowball sampling technique was used to recruit participants living in eastern North Carolina, an economically and geographically underserved area. This method relied upon information being shared by 'word of mouth.' We also reached our potential participants through two channels: 1) verbal advertisement at a local autism research fair for parents of children with ASD and 2) flyers sent to parents through the Autism Society of North Carolina, the local autism education agencies and Pitt County School District. Selection criteria used for participation stipulated that participants spoke either English or Spanish and were parents of at least one child diagnosed with ASD. Interested parents contacted the researchers directly and scheduled an appointment for individual face-to-face interviews.

\section{Procedures}

In order to protect confidentiality, participant information was coded using a numeric system. A master list of participants was kept in the office of the lead researcher (L.X.) in order to complete reliability checks during data analysis. Prior to the interview, the purpose of the study as well as the consent document for participation was reviewed and signed by each participant in their preferred language. Interviews lasted for 45-60 min each and a standard protocol of questions related to the study was used for all interviews. The bilingual specialist conducted the interviews for non-English speaking participants with one of the primary researchers present.

All interviews were audio recorded in order to provide a reliability check of information. Researchers also collected field notes during the interviews in order to ensure that all related information concerning the interview process, such as the participant's body language and tone, was recorded. Participants received a $\$ 40$ gift card for participation.

\section{Data analysis}

Each interview was transcribed verbatim and analyzed with the assistance of Nvivo 10 using a thematic analysis approach, which is an inductive method. The feature of this analytical approach was exploratory and theory-driven, rather than depending on pre-existing coding themes. The research team members met weekly to define and redefine the thematic categories as well as resolve discrepancies when diverging opinions arose in the course of data analysis. The researchers' triangulation (a method used to check and establish data validity via collecting multiple perspectives to arrive at consistency and uncover deeper meaning in the data) strengthened the reliability of our data.

\section{Results}

Over half of the 45 participants were mothers $(60 \%, n=27)$ and the remaining $(40 \%, \mathrm{n}=18)$ were fathers. Participants' mean age was 39.1 years $(\mathrm{SD}= \pm 4.9$, Range $=24-60$ years). More than half of our participants $(58 \%, \mathrm{n}=26)$ were part of a couple with another participant. The majority $(82 \%, n=37)$ of parents self-reported that they had one child with autism and the remaining reported they had more than one child with autism.

A large proportion of participants were White (58\%), followed by Hispanic (31\%), Black (9\%) and American Indian/Alaskan Native (2\%). With regard to annual income, slightly more than half of parents (53\%) had an annual income lower than $\$ 55 \mathrm{~K}, 42 \%$ reported their annual incomes was higher than $\$ 55 \mathrm{~K}$, and two did not respond to the item asking about their income.

Altogether, 44 children with ASD were involved in the study; 35 boys and 9 girls. The average age of the children was 8.8 years (range $=2-31$ ) and the average age of diagnosis was 4.4 years (range $=1.5-13$ ). The children were representative of varying severity levels of ASD as reported by parents.

\section{Interview finding}

Forty-five parents of children with autism were interviewed about their emotional responses towards CMA genetic testing. The researchers asked the parents the open-ended question "Do you think the CMA test may add extra stress or anxiety to your life, or maybe reduce your stress or anxiety? Can you explain why?" to elicit their emotional reactions to CMA testing. Based on parents' emotional reactions, the responses can be categorized into two broad subgroups: 1) parents with negative emotions (58\%) related to CMA genetic testing and 2) parents with positive emotions (42\%) associated with CMA genetic testing. Within each of these two categories, the reasons why they had or did not have negative emotions about CMA testing differed among participants and were thus further categorized as follows.

\section{Negative emotional reactions}

Figure 1 categorized the frequencies of the varied themes indicating parents' negative emotional reactions towards taking their children to undergo CMA genetic testing.

Fear, worry or anxiety: As indicated above, this is the most frequently mentioned theme indicated by seventeen participants. The most cited reason for this particular negative emotional reaction was associated with the uncertainty caused by the testing results. For instance, one participant claimed, "I think it would be the [test] results", she then added "Are you preparing me to tell me this news that's going to affect me and my child's life, for the rest of our lives? Or are you going to give me this news to say- no worries, you do not have - you're not 


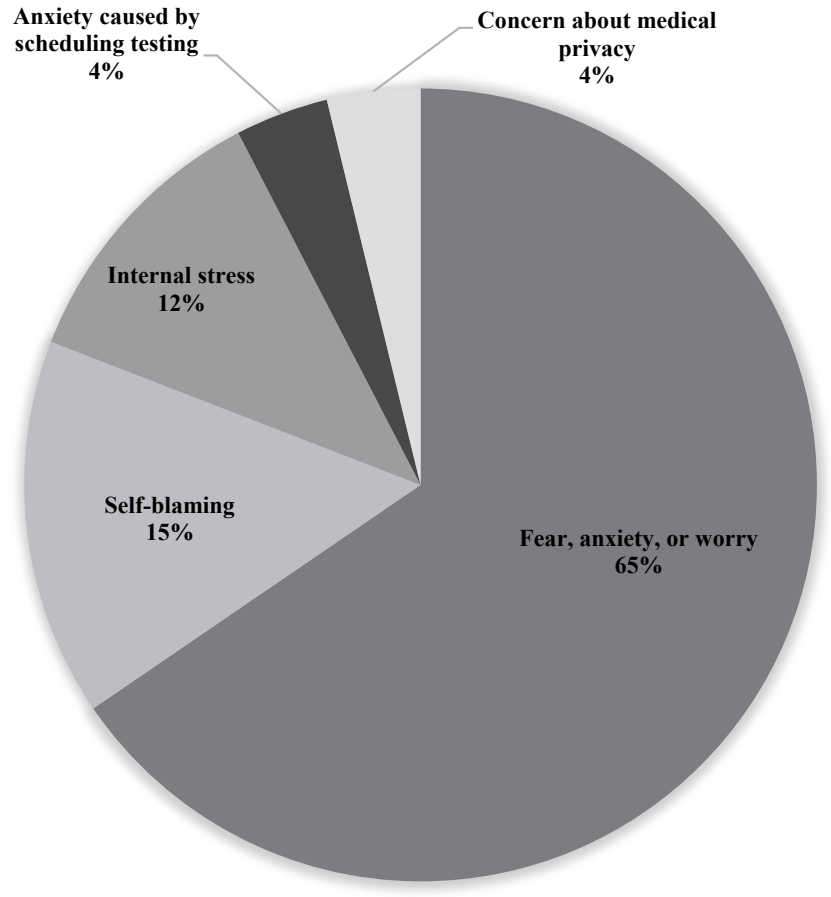

Figure 1: Frequencies of parents who had negative emotional responses towards CMA genetic testing $(n=26)$.

going to have to experience this emotional, life-long situation." This was echoed by another participant regarding the waiting time for the results who related, "It might take months for the test results to get back... and it could be nerve racking."

Self-blaming: Four participants expressed that either of the parents would feel guilty if the results indicated that they had passed on "bad genes" to their children. One father said, "My wife on the other hand did not want to have the emotional weight of feeling like she was the failure. She would rather identify that there was something wrong that was not her control."

Internal stress: Certain types of personal traits also caused three parents to have stress about the testing. One participant expressed straightforwardly that "emotionally I was a wreck" and the other, "that [testing] could probably cause some anxiety. We are more less that type of person [that easily get nervous]" Another participant stated he that he would think about it all the time if the test had been ordered but not yet completed.

Two parents said that getting the test might cause tension among family members. For instance, one mother said that she was most concerned that it would potentially cause fights or uneasiness if she decided to take his son to be tested, "I think it [undergoing the test] would add more stress and more chaos, I think internal chaos, especially."

Another two participants expressed that anxiety would come from their children who would undergo the test. As one mother remarked, "emotionally, it would not affect me. Children always have anxiety when they are dealing with testing because they do not know as much as we know". The other response was related to the concern about the child, "he has to know how something is going to happen...it is going to be very nervous just thinking about it and how to explain that to him and not being able to live a normal life."
Anxiety caused by scheduling testing: Due to the difficulty with reconciling the Scheduling, the genetic testing and work commitment caused one participant to have anxiety. The uneasiness caused by her rigid work schedule was expressed in her remarks: "It would with planning....about the flexibility...so with the [testing] scheduling kind a thing could add a little more stress from work which trickles down to him [son with autism] by me being less patient with him."

Concern about medical privacy: The confidentiality of medical records to other professionals became a concern to one parent. One mother said, "The medical records were all in one system the doctor could pull it up and he could see all the notes the neurologist made."

\section{Positive emotional reactions}

As demonstrated in Figure 2, parents who held positive emotional responses towards CMA genetic testing claimed different reasons for their favorable attitudes.

Alleviating stress: Eight interviewees thought that completion of the testing might alleviate their stress. For instance, some expressed that "it would lower stress and anxiety of knowing... better prepare us and I think that it would take away some of that constant worry of everything". Key words like "ease some concerns", "big relief", "they give the results to one, one prepares oneself mentally" are also among responses provided.

Confirming autism diagnosis: A specific relief that parents thought CMA genetic testing might bring to them is that it would help them relieve uncertainty about the cause of autism. Four people noted that they may feel relief brought on by confirming an autism diagnosis. One parent said "it would be less stressful, to know the motive or the causes". The eagerness to know the etiology of autism was found in a participant's response: "I would know why [he]...behaves like that, why he is like that. Or also, if it were genetic, the reason why he has it". Similarly, a parent had high confidence in CMA genetic testing because she thought CMA testing would help her get more information about her child with autism. She said, "We would take it with calm. It is something that would help us, maybe, for more information."

Parents' religious beliefs: The role of supernatural power in

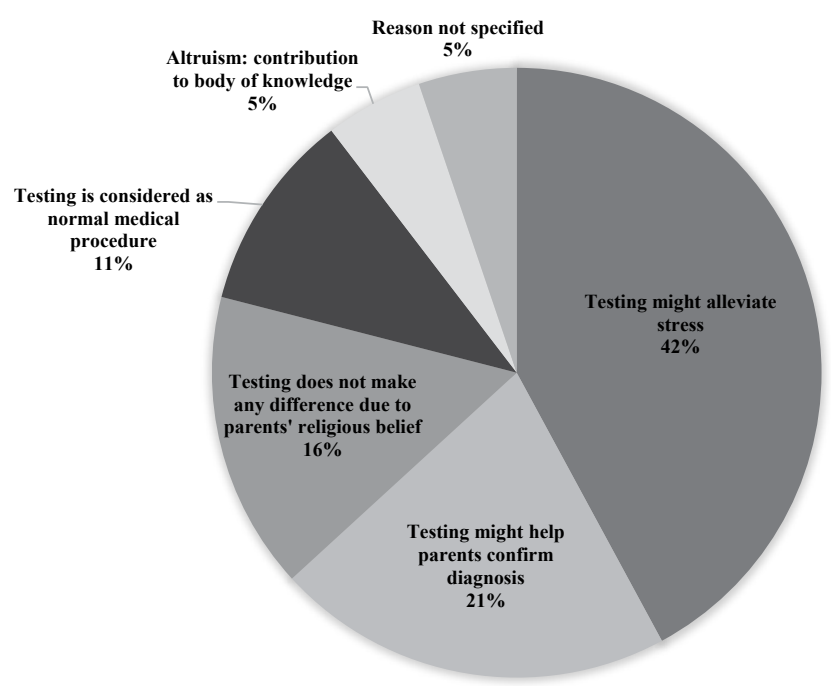

Figure 2: Frequencies of parents with positive emotional responses towards CMA genetic testing $(n=19)$. 
justifying the mental burden was mentioned by three parents. They all expressed the acceptance of having a child with autism because they believed God created the child. One participant remarked, "I do not think the test [CMA] can cause any distress, honestly, for myself, it is not going to change how much I love that child because God created that child and I...my job, is to love that child and to make that child as successful and as happy and as productive as I possibly can."

Normal medical procedure: Two participants held the belief that CMA is just a normal medical procedure among many others diagnostic or lab tests. These can be evidenced by the responses, "I do not think it [testing] would cause more anxiety, better still deal away with it" and "I do not think so because it [testing] is just something normal that happens in life."

Altruism: One parent showed altruism in her response to the interview question. She thought that providing genetic testing to children with autism would contribute to the body of knowledge regarding autism genetics as she stated, "This is something new that they provide a better resource and understanding to autism".

Reason not specified: There was one parent who did not specify a particular reason why CMA testing did not add extra stress or anxiety to her life. She just simply said "no" and refused to give further explanation about the reasons why she had no perceived emotional concerns.

\section{Discussion}

This pilot study allowed us to be the first to understand parents' perceived emotional responses to CMA-the newer generation genetic test for ASD prior to undergoing the test. In addition, the qualitative research methodology we adopted also enables us to maintain a dynamic and open conversation with the participants throughout our research process. Meanwhile, this research method rendered "ecological validity" with a focus on the authentic observations on the relationship of participants and responses of everyday tasks [16].

The most salient research finding is that over half of the participants (58\%) reported having negative emotions toward CMA genetic testing. Among these negative emotions, the feeling of fear, worry, anxiety, self-blaming and stress were the most prominent reported responses regardless of parents' educational level, income, and ethnicity. The underlying reasons that might contribute to parents' negative emotions towards CMA testing included lack of awareness of the test availability and test values, concerns about the high costs of testing as well as paucity of parental knowledge about the medical conditions of the affected children. Our findings were echoed by previous studies that had investigated parents' intention towards Fragile X genetic screening, which also indicated that anxiety, worry and fear might be the primary negative emotions associated with parents' decisions to take their children undergo the test [16]. Our findings also indicated that some negative emotions, such as fear or guilt might have a negative impact on peoples' intention to undergo CMA, as has been found with other genetic tests associated with autism among parents of children with at least one child diagnosed with ASD [17]. Considering a larger proportion of parents held negative emotions toward CMA genetic testing, pretest counseling that address parents' negative emotions would be highly recommended to dispel parents' concerns related to CMA.

This study measured a population in a rural area of Eastern North Carolina, where the percentage of people living in poverty is much higher than the rest of the state on average. Previous studies have found that distance and financial costs might be associated with participating in genetic research among families with affected members [18,19]. As an extension of these barriers, we believe there are certain correlations between negative emotional reactions and undergoing ASD genetic testing. As the first study of its kind, our research aims to explore parents' perceptions of CMA among community members and increase awareness regarding genetic testing for autism in underserved areas. More educational efforts need to be made regarding the availability of more advanced genetic tests for ASD-affected populations, pros and cons of testing, as well as how to make informed decisions based on the test results.

While the majority did not have a favorable perception of genetic testing, some parents in the study who held positive emotional responses to CMA testing viewed the test as a normal medical procedure with benefits in the process of care for a child with autism. This sample of parents is partially consistent with previous research on the clinical utility of genetic testing for autism, in which parents with favorable attitude are primarily concerned with its medical usefulness $[8,11,20]$. Caution is needed when we interpret parents' positive attitudes. Research has reported a paucity of understanding in those who have completed CMA genetic testing and how the test results can be applied $[14,21]$.

While CMA results can reliably substantiate a genetic basis or support known variants, results cannot always be ascertained as either 'negative,' meaning no identified genetic cause or 'pathogenic.' Nine percent of results will present with genetic 'variances of unknown significance' or VUS. These could either arise from de novo mutations or be present in the parent's genes and could either be benign or pathogenic [12]. Variations could also go undetected as VUS and tests concluded to be negative or pathogenic are not inerrant. If these parents were too optimistic about CMA genetic testing, negative findings may lead them to assume that a genetic causation has been ruled out. On the other hand, if the test results turned to have a genetic basis, this initially hopeful group of parents might have emotional reactions towards the results, like a heightened sense of guilt and self-blame for unknowingly passing on a potentially pathogenic genetic variant or may be disappointed in their prospects for clinical assistance [13] Ultimately, in clinical practice, counseling is needed to ensure that parents understand at length the implications of and options afforded by the test's completion, with their emotional wellbeing considered. At this point, there is a clear demand for both pre- and post-test counseling for families for whom the genetic testing process might be a psychologically dynamic experience. The ability to help parents understand the benefits of CMA with regards to its limitations may help ensure parents are soundly and accurately interpreting their results to receive the best possible outcome.

In addition, those parents who have unfavorable outcomes may dismiss or deny the results, and they may also experience heightened emotional trauma after the test. Lerman et al. [18] found that rates of depression in those who declined knowledge of test results of a similar nature increased from $26 \%$ pre-testing to $47 \%$ at one-month follow-up. The authors concluded that coping with risk by denial or avoidance may ultimately be detrimental. The accelerated emotions immediately after the test has been completed create an urgent need for post-test counseling for parents with guidance on how to cope with the negative psychological status when the testing results are discovered to be pathogenic [14]. Currently, there are not many professional genetic counselors in the field. It would be necessary that health education specialists and other professionals in the field of public health aid in the role of educational intervention for parents of children with autism that have the intention 
of undergoing CMA genetic testing. We need to devise educational interventions for parents with an emphasized goal of helping genetic testing candidates cope with negative emotions related to CMA testing.

\section{Limitations}

Our study inevitably suffered from some limitations. First, the diagnosis of children's autism and the severity level were based on the reports of parents, which were not officially verified by a professional document and made instead by parents' self-reported autism diagnosis. It is noteworthy that significant cultural differences in childhood experience and expectations for developmental behavioral norms can influence the detection and parental reporting of symptoms of autism $[22,23]$. The symptoms of autism reported was also subject to their own understanding that is shaped by the diversity of demographics, education level and socioeconomic status within our participation pool.

It may also be suggestive that factors in the participants' lives other than emotions have been demonstrated as impactful on decisions and attitudes towards genetic testing $[18,24,25]$. Ethnic populations with inherently stronger opinions about genetic testing or low-income parents who believe the costs of testing to be out of their possibilities of care may use these traits as a prevailing basis for their opinion [26]. If socioeconomic status or cultural biases had a decisive role in shaping personal beliefs about the test, this may result in individuals with inherent negative or positive attitudes about testing, which would lead to dismissive answers lacking in true consideration on their emotional states and particularly those of family members.

Of the utmost consideration is that all of the participants in this study were asked a question about their emotional responses to CMA genetic testing in a hypothetical sense without facing the real situation. In reality, the emotional convictions of parents would be much more intense when asked to make a well-rounded decision about undergoing CMA testing at a clinical visit. Our answers would also be different from that of if we had done an on-site interview with a participant about to go through the testing.

\section{Future Research Plans}

Future research could focus on the recruitment of a larger sample of parents with socially and economically disadvantaged backgrounds to fully address the needs of this population. It is our desire that future research can address the potential unmet needs that parents of lower socioeconomic status have due to financial or awareness barriers. If their financial concerns regarding CMA genetic testing which caused them to have emotional expressions were relieved, the research findings would otherwise present a different picture. The diverse demographic makeup in this area also poses a need for us to recruit more ethnic minorities in the future. Parents of African, Hispanic/Latino, Native and Asian American backgrounds are especially in need of evaluation and representation in order to comprehensively examine the needs of parents of children with ASD in diverse and underserved geographical areas. Lastly, future research should measure perceptions of parents who are offered CMA testing or who have just undergone testing.

\section{Acknowledgement}

We want to give special thanks to all the parents of children with ASD who participated in this pilot study.

\section{References}

1. American Psychiatric Association (2013) Diagnostic and statistical manual of mental disorders. 5th ed. Washington, DC: American Psychiatric Association.

2. Bauer SC, Msall ME (2011) Genetic testing for autism spectrum disorders 17:
$3-8$

3. Heil KM, Schaaf CP (2013) The genetics of autism spectrum disorders - A guide for clinicians. Curr Psychiatry Rep 15: 1-8.

4. Miller DT, Adam MP, Aradhya S (2010) Consensus statement: Chromosomal microarray is a first-tier clinical diagnostic test for individuals with developmental disabilities or congenital anomalies. Am J Hum Genet 86: 749-764.

5. McGrew SG, Peters BR, Crittendon JA, Veenstra-Vanderweele J (2012) Diagnostic yield of chromosomal microarray analysis in an autism primary care practice: Which guidelines to implement? J Autism Dev Disord 42: 1582-1591.

6. Vande Wydeven K, Kwan A, Hardan AY, Bernstein JA (2012) Underutilization of genetics services for autism: The importance of parental awareness and provider recommendation. J of Genet Couns 21: 803-813.

7. Schaefer GB, Mendelsohn NJ; Professional Practice and Guidelines Committee (2013) Clinical genetics evaluation in identifying the etiology of autism spectrum disorders: 2013 guideline revisions. Genet Med 15: 399-407.

8. Shen Y, Dies KA, Holm IA, Bridgemohan C, Sobeih MM, et al. (2010) Clinical genetic testing for patients with autism spectrum disorders. Pediatrics 125 727-735.

9. Miles JH (2011). Autism spectrum disorders--a genetics review. Genet Med 13 : 278-294.

10. Chen LS, Xu L, Huang TY, Dhar SU (2013) Autism genetic testing: A qualitative study of awareness, attitudes and experiences among parents of children with autism spectrum disorders. Genet Med 15: 274-281.

11. Narcisa V, Discenza M, Vaccari E, Rosen-Sheidley B, Hardan AY, et al (2013) Parental interest in a genetic risk assessment test for autism spectrum disorders. Clin Pediatr (Phila) 52: 139-146.

12. Reiff M, Bernhardt BA, Mulchandani S, Soucier D, Cornell D, et al (2012) "What does it mean?": Uncertainties in understanding results of chromosomal microarray testing. Genet Med 14: 250-258.

13. Reiff M, Giarelli E, Bernhardt BA, Easley E, Spinner NB, et al. (2015) Parents perceptions of the usefulness of chromosomal microarray analysis for children with autism spectrum disorders. J Autism Dev Disord 45: 3262-3275.

14. Giarelli E, Reiff M (2015) Mother's appreciation of chromosomal microarray analysis for autism spectrum disorders. J Spec Pediatr Nurs 20: 244-258.

15. Xu L, Lu W (2015) Emotional factors affecting Taiwanese parents' decisionmaking regarding autism genetic testing. Int J of Recent Sci Res 6: 4141-4148.

16. Xu L, Talwar D, Richman AR, Foster M (2015) A systematic review of emotions, attitudes and decisions regarding genetic tests associated with autism spectrum disorders. Int J Educ Soc Sci 2: 1-9.

17. Spooner DM, Pachana NA (2006) Ecological validity in neuropsychological assessment: A case for greater consideration in research with neurologically intact populations. Arch Clin Neuropsychol 21: 327-337.

18. Cuccaro ML, Czape K, Alessandri M, Lee J, Deppen AR, et al. (2014) Genetic testing and corresponding services among individuals with autism spectrum disorder (ASD). Am J Genet A 164: 2592-2600.

19. Hawkins AK, Hayden MR (2011) A grand challenge: Providing benefits of clinical genetics to those in need. Genet Med 13: 197-200.

20. Henderson LB, Applegate CD, Wohler E, Sheridan MB, Hoover-Fong J, et al. (2014) The impact of chromosomal microarray on clinical management: A retrospective analysis. Genet Med 16: 657-664.

21. Kiedrowski LA, Owens KM, Yashar BM, Schuette JL (2015) Parents' perspectives on variants of uncertain significance from chromosome microarray analysis. J Genet Couns 25: 101-111.

22. Lerman C, Hughes C, Lemon SJ, Main D, Snyder C, et al. (1998) What you do not know can hurt you: Adverse psychologic effects in members of BRCA1-linked and BRCA2-linked families who decline genetic testing. J Clin Oncol 16: 1650-1654.

23. Grinker R, Yeargin-Allsopp M, Boyle C (2011) Culture and autism spectrum disorders: The impact on prevalence and recognition in autism spectrum disorders. Oxford, UK: Oxford University Press.

24. Grinker RR, Chambers N, Njongwe N, Lagman A, Guthrie W, et al. (2012) "Communities" in community engagement: Lessons learned from autism research in South Korea and South Africa. Autism Res 5: 201-210.

25. Cragun D, Bonner D, Kim J, Narod SA, Gomez-Fuego A, et al. (2015) Factors 
Citation: Xu L, Sun L, Mitchell LC, Richman AR, Floyd AE (2016) Chromosomal Microarray Genetic Testing - A Qualitative Investigation among Parents of Children with Autism Spectrum Disorders in Rural North Carolina. J Clin Med Genomics 4: 143. doi: 10.4172/2472-128X.1000143

Page 6 of 6

associated with genetic counseling and BRCA testing in a population-based sample of young black women with breast cancer. Breast Cancer Res Trea 151: 169-176.
26. Zamora I, Williams ME, Higareda M, Wheeler BY, Levitt $P$, et al. (2016) Brie report: Recruitment and retention of minority children for autism research. $J$ Autism Dev Disord 46: 698-703. 